\title{
A Survey on Perspective about the Role of Robotics in Neurorehabilitation among Physiotherapists of Ahmedabad
}

\author{
Drashti Atulbhai Shah', Mihirdev P. Jhala \\ ${ }^{1}$ Second-Year MPT Student, JG College of Physiotherapy, Ahmedabad \\ ${ }^{2}$ Principal, JG College of Physiotherapy, Ahmedabad. \\ Corresponding Author: Drashti Shah
}

DOI: https://doi.org/10.52403/ijhsr.20220130

\begin{abstract}
Background: The world health organization estimates that approximately $15 \%$ of the world's population has a few forms of disability. Rehabilitation has a key role in reducing the level of disability. Application of robotics devices in rehabilitation help to achieve this goal. Robot-mediated neurorehabilitation is a growing and advanced field for treating neurological disease. The effectiveness of robotics therapy is ambiguous. Robotics therapy is a novice treatment in the physiotherapy profession and not that much-studied are executed in the field of robotics.

Aims and Objective: To determine the attitude towards the role of robotics in neurorehabilitation among physiotherapists of Ahmedabad.

Methodology: A cross-sectional observational study was conducted in 128 physiotherapists of Ahmedabad and snowball sampling was used. An online survey becomes executed with a self-reliant questionnaire. The questionnaire contains questions associated with perspectives about the role of robotics in neurorehabilitation. The statistical evaluation became accomplished with Microsoft Excel 2019.

Result: Our finding suggests that from the 128 subjects, $95(74 \%)$ were agreed robotics play important role in neurorehabilitation, 22(17\%) were Neutral, and 11(9\%) disagreed.

Conclusion: We found that most of the physiotherapists think robotics devices play important role in neurorehabilitation. $17 \%$ of physiotherapists are not sure and the rest of the physiotherapists think robotics devices do not play important role in neurorehabilitation.
\end{abstract}

Key Words: Neurorehabilitation robot, Qualified Physiotherapists, Ahmedabad

\section{INTRODUCTION}

Neurological dysfunction critically impacts the quality of life and may culminate in the inability to perform simple everyday activities. Unfortunately, such sensorimotor impairments are very frequent among neurological patients: More than two-thirds of all stroke patients have affected upper limbs ${ }^{[1]}$ and about $50 \%$ of them suffer from a chronic reduction in arm function ${ }^{[2]}$ These impairments can also affect the lower limb, compromising, with distinctive levels of severity, the sensorimotor strategies used by the brain during gait and balance control. To recover from those pathological conditions depends on the patient's specific impairment. For example, proprioceptive impairments affect motion planning ${ }^{[3,4]}$; paresis affects motion inaccuracy ${ }^{[5]}$; and abnormal muscle tone turns into loss of motion, smoothness, and intra-limb coordination. ${ }^{[6]}$

The evolution of rehabilitation robots began out in 1980. The following decade becomes a new phase. After the year 2000 , the primary representatives of 
commercially available robots appeared. These gadgets can help in the training of upper and lower limb movements and motor relearning, and in developing proprioception, cognitive functions, and attention. The application of robots in rehabilitation is not changing the therapist's work, but providing more treatment options. [7]

Robotic technology provides two key abilities (1) Examine the human sensorimotor function, and (2) re-education the human brain to enhance the patient's quality of life. ${ }^{[8]}$

Robotic devices for neurorehabilitation can be labeled into two major classes primarily based on the distinctive kind of physical human-robot interaction: end-effector devices and exoskeletons. An end-effector is an end of a device that attaches to a robot's wrist, allowing the robot to interact with its task. These systems do not control the entire kinematic chain. In this type of device, only possible to control the distal body segment that is connected to the end-effector. The exoskeleton controls the kinematics of the human limb and assists its actions via the location and the orientation of every joint. The gadgets are designed with the precise motive of coupling and aligning the mechanical joints to the human. ${ }^{[8]}$

Rehabilitation robots are used especially following central nervous system damage, usually after stroke. Multiple scientific trials and meta-analyses were achieved regarding those robots. Mehrholz et al. found that electromechanical armtraining promotes improvement in arm function and muscle strength, in addition to execution of activities of daily living. Nevertheless, the methodologies of the research were quite different, and 24 different devices were used. Robotmediated training on a treadmill is a widely used technique for gait re-education. ${ }^{[9]}$ Mehrholz et al. discovered that post-stroke patients who obtained robotics training in addition to conventional physiotherapy were more likely to achieve independent walking, than subjects who received conventional therapy alone. ${ }^{[10]}$

There are many robotics devices for the upper limb and lower limb was provided. The effectiveness of robotics therapy is ambiguous in the field of a physiotherapist, so the present study aims to find out perspectives about the role of robotics in neurorehabilitation among the physiotherapists of Ahmedabad.

\section{METHOD}

A cross-sectional observational study was conducted in 128 physiotherapists of Ahmedabad and snowball sampling was used. A self-reliant questionnaire was developed and spread through Google forms. The link of the questionnaire was sent through WhatsApp and other social media and the link was also shared with people apart from the first point of contact and so on. After they accepted to take part in the survey they fill up the demographic details and several questions related to the role of robotics in Neurorehabilitation.

Qualified Physiotherapists MPT Students, Clinicians, and Academicians, and people who were willing to participate were included. Those who are not able to understand English and subjects with any visual disabilities were excluded from the study.

The online self-reliant questionnaire was developed by the investigator. Item rated on 5-point Likert scale format. 5- point Likert Scale ranging from Strongly Disagree-1, Disagree-2, Neutral-3, Agree-4, Strongly Agree- 5. Scoring of questions: 11 to $19=$ Strongly Disagree, 20 to $28=$ Disagree, 29 to $37=$ Neutral, 38 to $46=$ Agree, 47 to $56=$ Strongly Agree. Statistical evaluation was performed with Microsoft Excel 2019.

\section{RESULTS}

The results show the descriptive character of the study, from 128 responses $87 \%$ were female and $13 \%$ were male. (Figure-1) 
Drashti Atulbhai Shah et.al. A survey on perspective about the role of robotics in neurorehabilitation among physiotherapists of Ahmedabad.

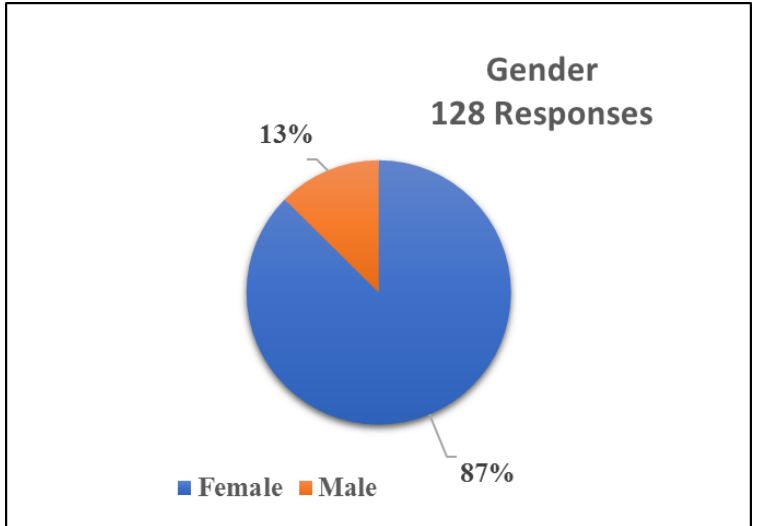

(Figure: 1 Gender distribution of the respondents)

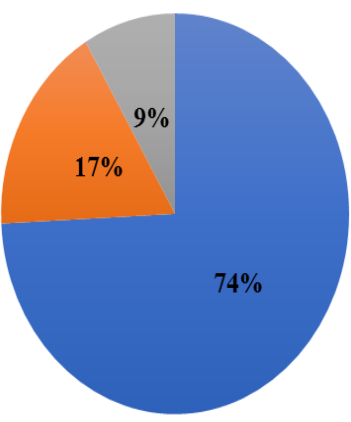

$\square$ Agree $\square$ Neutral $\square$ Disagree

Figure: 2 Scoring from Respondent.

We found from the 128 subjects, 95(74\%) were agreed, 22(17\%) were Neutral, and $11(9 \%)$ disagreed.

\begin{tabular}{|l|l|l|l|l|l|}
\hline Question & \multicolumn{1}{|l}{ Table 1: Question Wise Analysis } \\
\hline $\begin{array}{l}\text { Disongly } \\
\text { Disagree }\end{array}$ & Disagree & Neutral & Agree & $\begin{array}{l}\text { Strongly } \\
\text { Agree }\end{array}$ \\
\hline $\begin{array}{l}\text { 2) Do you think robot-mediated neurorehabilitation is a growing and advanced } \\
\text { method in treating neurological conditions. }\end{array}$ & $10(8 \%)$ & $12(9 \%)$ & $11(9 \%)$ & $80(63 \%)$ & $22(8 \%)$ \\
\hline $\begin{array}{l}\text { 3) Do you think neurorehabilitation robots reported good comfort, } \\
\text { acceptability, and satisfaction. }\end{array}$ & $28(22 \%)$ & $23(18 \%)$ & $35(27 \%)$ & $56(44 \%)$ & $10(22 \%)$ \\
\hline $\begin{array}{l}\text { 4) Do you think robotics should be made accessible to both rural and urban } \\
\text { communities. }\end{array}$ & $14(11 \%)$ & $25(20 \%)$ & $27(21 \%)$ & $39(30 \%)$ & $28(11 \%)$ \\
\hline $\begin{array}{l}\text { 5) Do you think robotics devices improve upper limb and lower limb function } \\
\text { in a shorter time compared to conventional protocol. }\end{array}$ & $11(9 \%)$ & $19(15 \%)$ & $37(29 \%)$ & $53(41 \%)$ & $14(9 \%)$ \\
\hline $\begin{array}{l}\text { 6) Do you think the anxiety of patients for machines can be a negative factor } \\
\text { in robotic therapy. }\end{array}$ & $23(18 \%)$ & $23(18 \%)$ & $29(23 \%)$ & $60(47 \%)$ & $11(18 \%)$ \\
\hline $\begin{array}{l}\text { 7) Do you think lack of operational and technological knowledge affects the } \\
\text { benefits of robotics in neurorehabilitation. }\end{array}$ & $35(27 \%)$ & $10(8 \%)$ & $10(8 \%)$ & $82(64 \%)$ & $23(27 \%)$ \\
\hline 8) Do you think neuro robotics should be made affordable? & $9(7 \%)$ & $12(9 \%)$ & $15(12 \%)$ & $59(46 \%)$ & $35(7 \%)$ \\
\hline 9) Do you recommend your patients opt for neurorobotics? & $10(8 \%)$ & $20(16 \%)$ & $28(22 \%)$ & $65(51 \%)$ & $9(8 \%)$ \\
\hline $\begin{array}{l}\text { 10) Do you think that robotics devices provide a good experience along with } \\
\text { recovery to the patient, unlike conventional protocols. }\end{array}$ & $46(36 \%)$ & $26(20 \%)$ & $38(30 \%)$ & $51(40 \%)$ & $10(36 \%)$ \\
\hline $\begin{array}{l}\text { 11) Do you think workshops and seminars should be done towards knowledge } \\
\text { about robotics in neurorehabilitation. }\end{array}$ & $5(4 \%)$ & $3(2 \%)$ & $8(6 \%)$ & $65(51 \%)$ & $46(4 \%)$ \\
\hline
\end{tabular}

\section{DISCUSSION}

The frequency of neurological problems in India provides a rough estimate of over 30 million people with neurological problems (excluding neuro infections and traumatic injuries). Higher rates of prevalence of neurological problems in rural areas, 6-8 million people with epilepsy, and high case fatality rates of stroke (27$42 \%){ }^{[11]}$

Robotic gadgets are well-applicable to help in this area, primarily based on their capacity to perform, repetitive tasks with consistency. Robots may be programmed to guide a patient through a series of specific motions while maintaining a prescribed degree of help and limiting undesired movements. Robots are also able to perform repetitive movements without fatiguing, while simultaneously collecting objective quantitative data. robotic gadgets provide patient engagement throughout repetitive physical tasks that can be tough to achieve throughout conventional exercise therapy. [12]

Nowadays, research on the use of robotic devices in various fields of healthcare systems is outstretched. ${ }^{[13-15]}$ In the field of rehabilitation, scientific literature shows numerous classifications of such systems consistent with their level of interaction ${ }^{[16]}$, the upper limb and lower limb that are treated ${ }^{[17-20]}$, and the potency of treatment ${ }^{[21-24]}$. Robotics therapy is a novice treatment in the physiotherapy profession and not that much-studied are executed in the field of robotics. 
In the present study to find out perspective about the role of robotics in neurorehabilitation among physiotherapists of Ahmedabad. We found that most physiotherapists think robotics devices play important role in neurorehabilitation. $17 \%$ of physiotherapists are not sure and the rest of the physiotherapists think robotics devices do not play important role in neurorehabilitation.

We found that $57 \%$ of the qualified physiotherapists of Ahmedabad agreed robotics devices play a major role in neurorehabilitation. Loris Pignolo et al. found that robotic rehabilitation induced a significant improvement in stroke patients [25]. $63 \%$ of physiotherapists agreed with robot-mediated neurorehabilitation is a growing and advanced method in treating neurological conditions. $44 \%$ of physiotherapists agreed with neurorehabilitation robots provide good comfort, acceptability, and satisfaction. $30 \%$ of physiotherapists agreed robotics should be made accessible to both rural and urban communities. $41 \%$ of physiotherapists agreed robotics devices improve upper limb and lower limb function in a shorter time compared to conventional protocol. Bryan ping ho chung found that robotic-assisted gait training improves ambulation and balance in stroke patients. ${ }^{[26]}$

$47 \%$ of physiotherapists agreed to the anxiety of patients for machines can be a negative factor in robotic therapy. $64 \%$ of physiotherapists agreed lack of operational and technological knowledge affects the benefits of robotics in neurorehabilitation. $51 \%$ of physiotherapists agreed to suggest their patients for neurorobotics treatment. $40 \%$ of physiotherapists agreed that robotics devices provide a good experience along with recovery to the patient, unlike conventional protocols. Alexa Keeling et al designed robotic upper extremity therapy tasks in subacute stroke patients and found that robotics therapy showed potential to improve outcomes in subacute stroke. ${ }^{[27]}$

$$
\text { Ledycnarf Holanda concluded }
$$
robotic devices as an innovative and effective therapy for the rehabilitation of individuals with SCI. ${ }^{[28]}$ Yu-ping Chen et al. found that robotic therapy improves upper extremity function in children with cerebral palsy. ${ }^{[29]}$ Ksenia Ustinova et al conducted a case report and found that robotic therapy improves the function of bradykinesia, rigidity, freezing, leg agility, gait, and posture in Parkinson's patients. ${ }^{[30]}$

The study has several limitations. The study was done in qualified physiotherapists MPT Students, Clinicians, and Academicians, so that result was not focused on any particular field of physiotherapists. The proportion of gender distribution is not equal. The future recommendation of the study is that it can be conducted in different states of India.

\section{CONCLUSION}

Our finding revealed that from 128 respondents most of the physiotherapists think robotics devices play important role in neurorehabilitation. $17 \%$ of physiotherapists are not sure and the rest of the physiotherapists think robotics devices do not play important role in neurorehabilitation.

Hence, qualified physiotherapists should design seminars and workshops related to the role of robotics in neurorehabilitation that will help to provide knowledge to undergraduate physiotherapist students.

\section{Acknowledgement: None}

\section{Conflict of Interest: None}

\section{Source of Funding: None}

\section{Ethical Approval: Approved}

\section{REFERENCES}

1. Hatem SM, Saussez G, della Faille, et al. Rehabilitation of motor function after stroke: A multiple systematic review focused on techniques to stimulate upper extremity recovery. Front. Hum. Neurosci. 2016, 10, 442 . 
2. Broeks JG, Lankhorst GJ, Rumping $\mathrm{K}$, et al.The long-term outcome of arm function after stroke: Results of a follow-up study. Disabil. Rehabil. 1999, 21, 357-364.

3. Coderre AM, Zeid AA, Dukelow SP et al. Assessment of upper-limb sensorimotor function of subacute stroke patients using visually guided reaching. Neurorehabil. Neural Repair 2010, 24, 528-541.

4. Winward CE, Halligan PW, Wade DT. Current practice and clinical relevance of somatosensory assessment after stroke. Clin. Rehabil. 1999, 13, 48-55.

5. Lang CE, Bland MD, Bailey RR. Assessment of upper extremity impairment, function, and activity after stroke: Foundations for clinical decision making. J. Hand Ther. 2013, 26, 104-115.

6. Lang CE, Beebe JA. Relating movement control at upper extremity segments to loss of hand function in people with chronic hemiparesis. Neurorehabil. Neural Repair 2007, 21, 279-291

7. Poli P, Morone G, Rosati G, et al. Robotic technologies and rehabilitation: new tools for stroke patients' therapy. Biomed Res Int. 2013; 153872.

8. Riccardo I, Francesca M, Marianna S et al. Perspectives and Challenges in Robotic Neurorehabilitation. Appl. Sci. 2019, 9, 3183

9. Mehrholz J, Pohl M, Platz $\mathrm{T}$, et al. Electromechanical and robot-assisted arm training for improving activities of daily living, arm function, and arm muscle strength after stroke. Cochrane Database Syst Rev. 2018 Sept 3;9:CD006876.

10. Mehrholz J, Thomas S, Werner C, et al. Electromechanical-assisted training for walking after stroke. Cochrane Database Syst Rev. 2017 May;10(5): CD006185.

11. M.Gourie -Devi, Epidemiology of neurological disorders in India: Review of background, prevalence and incidence of epilepsy, stroke, Parkinson's disease, and tremors. 2014, 62,6, 588-598

12. Weber, Lynne M., et al., The use of robots in stroke rehabilitation: A narrative review. NeuroRehabilitation. 2018,43,99-110.

13. K. S. G. Chua and C. W. K. Kuah, "Innovating with rehabilitation technology in the real world: promises, potentials, and perspectives," American Journal of Physical Medicine \& Rehabilitation, 2017,96(10), S150-S156.
14. H. M. Van der Loos, D. J. Reinkensmeyer, and E. Guglielmelli, "Rehabilitation and health care robotics," in Springer Handbook of Robotics, B. Siciliano and O. Khatib, Eds., pp. 1685-1728, Springer International Publishing, Cham, 2016.

15. Gomes P, "Surgical robotics: reviewing the past, analyzing the present, imagining the future," Robotics and Computer-Integrated Manufacturing, 2011, 27(2)261-266,

16. Huang H, Wolf SL, and J. He, "Recent developments in biofeedback for neuromotor rehabilitation," Journal of Neuroengineering and Rehabilitation. 2006,3(1)

17. Poli P, Morone GR, and Masiero S, "Robotic technologies and rehabilitation: new tools for stroke patients' therapy," BioMed Research International, 2013.

18. Timmermans AA, Seelen HA, Willmann $\mathrm{RD}$, et al, "Technology-assisted training of arm-hand skills in stroke: concepts on reacquisition of motor control and therapist guidelines for rehabilitation technology design," Journal of Neuroengineering and Rehabilitation, 2009, 6(1)

19. Diaz I, Gil JJ, and Sanchez E, "Lower-limb robotic rehabilitation: literature review and challenges," Journal of Robotics, 2011.

20. X. Lv and Z. Wu, "Review of robot-assisted gait rehabilitation after stroke," Journal of Rehabilitation Robotics, 2013, 3-8.

21. C. Lo, Guarino PD, Richards LG et al., "Robot-assisted therapy for long-term upper-limb impairment after stroke," New England Journal of Medicine, 2010,362(19), 1772-1783.

22. Kwakkel G, Kollen BJ, and Krebs HI, "Effects of robot-assisted therapy on upper limb recovery after stroke: a systematic review," Neurorehabilitation and Neural Repair, 2008,22(2)111-121.

23. Norouzi-Gheidari N, Archambault PS, and Fung, "Effects of robot-assisted therapy on stroke rehabilitation in upper limbs: systematic review and meta-analysis of the literature," Journal of Rehabilitation Research and Development, 2012, 49(4)479-496,

24. Veerbeek JM, Langbroek-Amersfoort et al. "Effects of robot-assisted therapy for the upper limb after stroke: a systematic review and metaanalysis," Neurorehabilitation and Neural Repair, 2017,31(2),107-121. 
Drashti Atulbhai Shah et.al. A survey on perspective about the role of robotics in neurorehabilitation among physiotherapists of Ahmedabad.

25. Loris P, Sebastiano et al. Data on a new neurorehabilitation approach targeting functional recovery in stroke patients. Data in brief 27(2019). https://doi.org/ 10.1016/j.dib.2019.104685

26. Chung BPH. Effectiveness of roboticassisted gait training in stroke rehabilitation: A retrospective matched control study. Hong Kong Physiother J. 2016 Nov 9;36: 10-16. doi: 10.1016/j.hkpj.2016.09.001. PMID: 30931034; PMCID: PMC6385094.

27. Alexa B. Keeling et al. Robot enhanced stroke therapy optimizes rehabilitation: a pilot study. Journal of NeuroEngineering and rehabilitation. 10 (2021)

28. Ledycnaf Holanda et al. Robotic assisted gait as tool for rehabilitation of individuals with spinal cord injury: a systematic review. Journal of Neuroengineering and rehabilt 126, 2017.

29. Yu-ping chen et al. Effects of robotic therapy on upper-extremity function in children with cerebral palsy: A systemic review. Dev Neurorehabil. 2016; 19(1): 6471.

30. Ksenia Ustinova et al. Effect of robotic locomotor training in an individual with Parkinson's disease: a case report. Disability and Rehabilitation.2011; 6(1): 77-85

How to cite this article: Shah DA, Jhala MP. A survey on perspective about the role of robotics in neurorehabilitation among physiotherapists of Ahmedabad. Int J Health Sci Res. 2022; 12(1): 223-228. DOI: https://doi.org/10.52403/ijhsr. 20220130 